\title{
Baicalin inhibits the metastasis of highly aggressive breast cancer cells by reversing epithelial-to-mesenchymal transition by targeting $\beta$-catenin signaling
}

\author{
TAO ZHOU ${ }^{1,2^{*}}$, AIJIE ZHANG $^{1,2^{*}}$, GE KUANG $^{2}$, XIA GONG $^{3}$, RONG JIANG $^{4}$, DAN LIN ${ }^{2}$, JIE LI $^{1,2}$, \\ HONGZHONG LI ${ }^{5}$, XIANG ZHANG ${ }^{1}$, JINGYUAN WAN ${ }^{2}$ and HONGYUAN LI ${ }^{1}$ \\ ${ }^{1}$ Department of Endocrine and Breast Surgery, The First Affiliated Hospital of Chongqing Medical University; \\ ${ }^{2}$ Chongqing Key Laboratory of Biochemistry and Molecular Pharmacology, Chongqing Medical University; \\ ${ }^{3}$ Department of Anatomy, Chongqing Medical University; ${ }^{4}$ Laboratory of Stem Cell and Tissue Engineering, \\ Chongqing Medical University; ${ }^{5}$ Molecular Oncology and Epigenetics Laboratory, The First Affiliated Hospital \\ of Chongqing Medical University, Chongqing 400016, P.R. China
}

Received February 14, 2017; Accepted July 28, 2017

DOI: 10.3892/or.2017.6011

\begin{abstract}
Metastasis is the main cause of death in breast cancer patients, which is due partly to the lack of effective treatment. Baicalin, a flavonoid compound isolated from the roots of Scutellaria lateriflora Georgi (Huang Qin), has recently been confirmed as an effective agent for the treatment of a variety of cancers. Yet, the effects and underlying molecular mechanisms of baicalin in regards to the metastasis of breast cancer remain unclear. In the present study, we found that baicalin had the potential to suppress the migration and invasion of highly aggressive breast cancer cells in a dose-dependent manner but had no impact on the viability of these cancer cells. Additionally, baicalin reversed the epithelial-to-mesenchymal transition (EMT) process, as evaluated by EMT markers in breast cancer cell lines with a change from a mesenchymal feature to an epithelial type. At the same time, the expression of $\beta$-catenin mRNA and protein was dose-dependently downregulated by baicalin in highly invasive breast cancer cell lines, and overexpression of $\beta$-catenin by adenoviruses abolished these beneficial effects of baicalin in regards to the migration and invasion, and EMT of breast cancer cells. Furthermore, using a xenograft mouse model, baicalin markedly reduced
\end{abstract}

Correspondence to: Dr Hongyuan Li, Department of Endocrine and Breast Surgery, The First Affiliated Hospital of Chongqing Medical University, 1 Youyi Road, Chongqing 400016, P.R. China E-mail: hongy_li@hotmail.com

Dr Jingyuan Wan, Chongqing Key Laboratory of Biochemistry and Molecular Pharmacology, Chongqing Medical University, Chongqing 400016, P.R. China

E-mail: jywan@cqmu.edu.cn

*Contributed equally

Key words: epithelial-mesenchymal transition, baicalin, breast cancer, metastasis, $\beta$-catenin liver and lung metastasis of breast cancer, inhibited expression of $\beta$-catenin, and degraded the EMT molecules vimentin and Slug in the orthotopic tumor tissues. Taken together, all these results indicate that baicalin effectively suppresses the metastasis of breast cancer by reversing EMT, which may be mediated by downregulation of $\beta$-catentin expression.

\section{Introduction}

Breast cancer is the most frequently diagnosed cancer and the leading cause of cancer-related death among women, and is a major health concern for women around the world. There were an estimated 1.7 million new cases $(25 \%$ of all cancer cases in women) and 0.5 million breast cancer deaths $(15 \%$ of all cancer-related deaths in women) globally in females in 2012 (1). In China, among the 5 most commonly diagnosed cancers, breast cancer alone accounted for $15 \%$ of the cases (2). Although there are numerous adjuvant treatments with new drugs and systematic treatment schedules to be applied in the clinic, metastasis, the mainly cause of death in breast cancer patients partly due to the lack of effective treatment, is still a huge barrier to overcome for therapy (3). Thus, to identify agents which can effectively inhibit breast cancer metastasis and explore the related mechanisms may provide a solution for this issue.

Epithelial-to-mesenchymal transition (EMT), which is involved in embryonic development, reconstruction of wounded tissues and fibrosis, plays an important role in tumor formation and metastasis (4). EMT is a process that guides the transformation of adhesive, non-mobile, polar, epitheliallike tumor cells into cells with a mobile, invasive, non-polar mesenchymal-like phenotype, which provides the potential for tumor cells to migrate to distant sites and form metastatic tumors. During this process, molecular biomarkers of epithelial cells such as E-cadherin and claudin are downregulated while markers of mesenchymal cells including vimentin and $\mathrm{N}$-cadherin are altered in an opposite way (5). A series of transcriptional factors (TFs) have been identified as EMT 
regulators, directly or indirectly repressing the encoding of E-cadherin. These TFs such as ZEB, Snail, Slug, Twist, are regulated by various complex signaling pathway networks such as Hedgehog $(\mathrm{Hh}), \mathrm{Wnt} / \beta$-catenin, Notch, or transforming growth factor- $\beta$ (TGF- $\beta$ ), by acting as isolated units or crosstalking to provide tumor cells with an additional mechanism with which to escape the effects of chemotherapy (6).

In recent years, due to the resistance of tumor cells to traditional therapy, dietary chemopreventive agents, as additional treatment strategies against cancers, have received increased attention for their excellent effects to suppress, reverse, or retard the process of tumorigenesis (7). Baicalin (Fig. 1A), a flavonoid compound isolated from the roots of Scutellaria baicalensis Georgi, has been demonstrated to possess many different pharmacological actions including antioxidant, anti-inflammation, anti-HIV-1 and antitumor activity (8-12). In regards to the antitumor activities, accumulating evidence reveals that baicalin exhibits its function in a wide range of cancers such as hematological malignancies, hepatic cancer, gallbladder carcinoma, lung cancer, colorectal cancer, breast cancer and bladder cancer (13-19), by inducing cell apoptosis, cycle arrest and autophagy, suppressing cell proliferation and tumor growth, and inhibiting migration, invasion and metastasis of cancer cells $(15,18,20,21)$. Although research has reported the protective activities of baicalin in malignancies, its underlying detailed mechanisms and its relationship with EMT remain unclear. Furthermore, the upstream intracellularsignaling cascades of metastatic breast cancer cells with high malignancy remain indeterminate.

MDA-MB-231 is an estrogen-independent fibroblastic human triple-negative breast cancer cell line lacking the estrogen receptor (ER), progesterone receptor (PR) and human epidermal growth factor receptor (HER)-2 (22). 4T1 is a mouse mammary cancer cell line, possessing the characteristic of resistance to 6-thioquanine and metastasizes from the primary tumor to multiple distant sites spontaneously including lymph nodes, blood, liver and lung (23). In the present study, we selected these two highly aggressive breast cancer cell lines to investigate the potential effects and mechanisms of baicalin on the metastasis of breast cancer in vitro and in vivo.

\section{Materials and methods}

Reagents and antibodies. Baicalin (purity $>99 \%$ ) was obtained from Zelang Medical Technology Co. (Nanjing, China). Crystal violet was purchased from Sigma (St. Louis, MO, USA). Vectastain ABC kit and liquid DAB+ Substrate Chromogen system for immunohistochemistry were purchased from Vector Laboratories Inc. (Burlingame, CA, USA) and Dako (Carpinteria, CA, USA), respectively. RPMI-1640 medium, Dulbecco's modified Eagle's medium (DMEM), trypsin-EDTA, phosphate-buffered saline (PBS) and penicillin/streptomycin were the products of Hyclone Laboratories Inc. (Los Angeles, CA, USA). Fetal bovine serum (FBS) was purchased from Gibco (Grand Island, NY, USA). Antibodies used in this study were anti-E-cadherin, anti-claudin, anti-vimentin, anti-Ncadherin, anti- $\beta$-catentin, anti-Snail and anti-Slug from Cell Signaling Technology (New England Biolabs, Ipswich, MA, USA); anti-Ki67 (rabbit monoclonal), secondary antibody (rabbit monoclonal IgG) from Abcam (Cambridge, UK); anti-
GAPDH from Santa Cruz Biotechnology (Santa Cruz, CA, USA); anti-F-actin-Green 488, and Alexa Fluor 594 (goat-antirabbit IgG) from Molecular Probes (Oregon, Eugene, OR, USA).

Cell lines and culture. Human breast cancer cell line MDA-MB-231 and mouse mammary cancer cell line 4T1 were obtained from the American Type Culture Collection (ATCC; Manassas, VA, USA) and cultured in DMEM and RPMI-1640 medium containing $10 \%$ FBS and $1 \%$ penicillin/streptomycin, respectively. Both of the cell lines were cultured in a humidified atmosphere with $5 \% \mathrm{CO}_{2}$ at $37^{\circ} \mathrm{C}$.

Cell viability assay. Cell viability was determined by crystal violet assays. MDA-MB-231 and 4T1 breast cancer cells were harvested with $0.05 \%$ trypsin-EDTA and seeded in 24-well plates (Corning, MA, USA) at a density of 6x10 $/$ well and $1 \times 10^{6} /$ well, respectively. Four hours later, the cells were treated with different concentrations of baicalin $(10,30$, or $100 \mu \mathrm{M})$ dissolved in fresh medium or vehicle, and incubated for another $72 \mathrm{~h}$. Then cells were fixed with $10 \%$ formaldehyde solution and stained with $0.25 \%$ crystal violet. After being washed with PBS, crystal violet was dissolved and the absorbance was read at $570 \mathrm{nM}$ using a microplate reader (ELx800; BioTek, Winooski, VT, USA).

Wound healing assay. MDA-MB-231 and 4T1 breast cancer cells were seeded in 6-well plates (Corning, MA, USA) at a density of $2 \times 10^{5} /$ well. Scrapes were made over the cells by a sterile toothpick until cells formed a confluent monolayer. After scraping, the cells were washed with PBS twice, and then replaced with fresh medium with or without various concentrations of baicalin and continued to culture. The width of the wound was monitored and photographed using a microscope (Nikon, Tokyo, Japan) at 100-fold magnification until the wound of the control group healed or almost healed.

Transwell migration assay. The migration assay was performed in 24-well Transwell chambers (Corning, MA, USA). Briefly, complete DMEM with $10 \%$ FBS with or without various concentrations of baicalin was added into the lower chambers, and MDA-MB-231 $\left(10^{5}\right)$ and 4T1 breast cancer $\left(6 \times 10^{4}\right)$ cells suspended in serum-free DMEM were added into the upper chambers. After incubation for $18 \mathrm{~h}$, the cells were fixed with $10 \%$ formaldehyde solution and stained with $0.25 \%$ crystal violet. Then the non-migration cells on the upper chamber were wiped off and the migrated cells in the lower chamber were photographed under a microscope (Nikon) at 100-fold magnification.

Immunofluorescence (IF) analysis. MDA-MB-231 and 4T1 breast cancer cells were seeded in 24-wells and treated with $100 \mu \mathrm{M}$ baicalin for $24 \mathrm{~h}$. After being fixed with $4 \%$ paraformaldehyde and permeabilized with $0.05 \%$ Triton X-100 at room temperature, the cells were incubated with primary antibodies anti-vimentin (1:100) or anti-Slug (1:50) overnight at $4^{\circ} \mathrm{C}$. After a further wash with PBS, the cells were incubated with Alexa Fluor 594-conjugated secondary antibody (dilution 1:500) for $1 \mathrm{~h}$. Then, anti-F-actin-Green 488 (dilution 1:500) was added to stain the cytoskeleton and the nuclei were stained 
by DAPI. Images were captured at x200 under a fluorescence microscope (Nikon).

Immunohistochemical (IHC) analysis. Tissues were fixed, dehydrated, embedded and cut into 5- $\mu \mathrm{m}$ sections in accordance with standard procedures. After deparaffinization, rehydration, antigen retrieval and blockage, the sections were incubated with primary antibodies overnight at $4^{\circ} \mathrm{C}$. Then the sections were blocked the endogenous peroxidase with hydrogen peroxide and incubated with the biotinylated goat anti-rabbit secondary antibodies (1:100). After signal amplification with avidin and horseradish peroxidase (HRP)-conjugated biotin, the sections were counterstained with DAB to visualize the nuclei. Finally, the sections were mounted and imaged.

Protein isolation and western blot analysis. Cells were lysed in cell lysis buffer containing various enzyme-protecting agents to collect total proteins. The concentration of protein was determined by the BCA kit. Total proteins $(40 \mu \mathrm{g})$ were separated on $10 \%$ SDS gel and transferred onto polyvinylidene fluoride (PVDF) membranes. After blocked with 5\% BSA in Tris-buffered saline (TBS) containing $0.1 \%$ Tween-20, the membranes were incubated with the primary monoclonal antibody at $4{ }^{\circ} \mathrm{C}$ overnight. The membranes were washed with TBST three times, followed by incubation with the secondary antibodies labeled with horseradish peroxidase (HRP). Finally, the membranes were visualized with an enhanced chemiluminescent system.

Quantitative reverse transcription-polymerase chain reaction (qRT-PCR). Total RNA from cancer cells was extracted by using the RNA isolation kit according to the manufacturer's instructions, and cDNA was synthesized using PrimeScript RT reagent kit with $1 \mu \mathrm{g}$ total RNA. qPCR was performed by using the PCR kit according to the instructions. The following primer sequences were used: E-cadherin sense, 5'-TCCTGGG CAGAGTGAATTTTGAAGA-3' and antisense, 5'-AAACGG AGGCCTGATGGGG-3'; claudin sense, 5'-CCTCCTGGGA GTGATAGCAAT-3' and antisense, 5'-GGCAACTAAAATA GCCAGACCT-3'; vimentin sense, 5'-TACAGGAAGCTGCT GGAAGG-3' and antisense, 5'-ACCAGAGGGAGTGAATC CAG-3'; N-cadherin sense, 5'-AGCCAACCTTAACTGAGG AGT-3' and antisense, 5'-GGCAAGTTGATTGGAGGG ATG-3'; Snail sense, 5'-TCGGAAGCCTAACTACAGCGA-3' and antisense, 5'-AGATGAGCATTGGCAGCGAG-3'; Slug sense, 5'-GGGGAGAAGCCTTTTTCTTG-3' and antisense, 5'-TCCTCATGTTTGTGCAGGA G-3'; and GAPDH sense, 5'-TGTTGCCATCAATGACCCCTT-3' and antisense, 5'-CTCCACGACGTACTCAGC G-3'. Relative quantification was achieved by normalization to GAPDH.

Xenograft model. Six- to eight-week-old female BALB/c mice were obtained from the Laboratory Animal Center of Chongqing Medical University and housed in a specific pathogen-free (SPF) laboratory environment. BALB/c mice were injected subcutaneously with $1 \times 10^{6} 4 \mathrm{~T} 1$ cells into the bilateral gluteal region. One week after inoculation, the mice were divided randomly into a sham-treated group and a baicalin-treated group. The former received PBS while the latter received $100 \mathrm{mg} / \mathrm{kg}$ baicalin in an intraperitoneal
A<smiles>O=C(O)C1O[C@H](Oc2cc3oc(-c4ccccc4)cc(=O)c3c(O)c2O)[C@H](O)[C@H]1O</smiles>

B

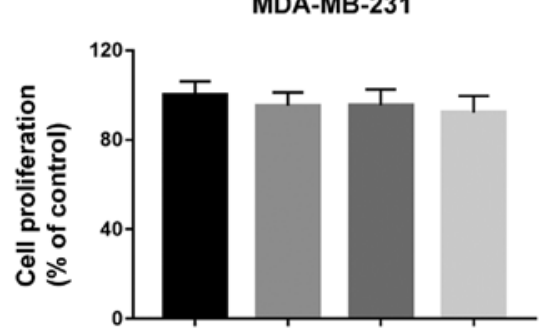

C

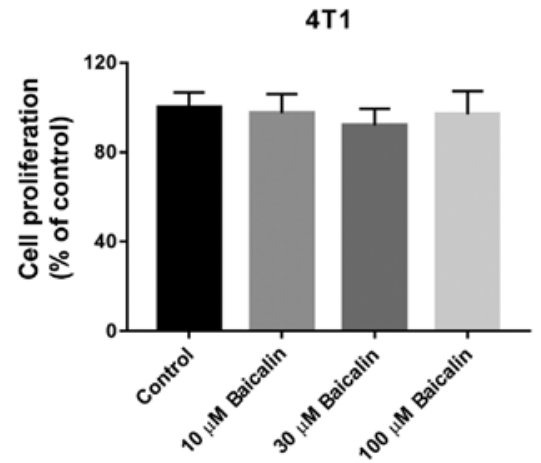

Figure 1. Baicalin does not affect the viability of breast cancer cells in vitro. The highly invasive breast cancer cells were pretreated with different concentrations of baicalin for $72 \mathrm{~h}$. (A) Chemical structure of luteolin. The viability of (B) MDA-MB-231 and (C) 4T1 cells was assayed by crystal violet assay. The results are shown as the mean $\pm \operatorname{SD}(n=3)$.

injection approach every 3 days. After 6 weeks, all mice were sacrificed under anesthesia, the tumors and lungs were excised, weighed, counted for tumor nodules and fixed for further analysis.

Statistical analysis. All data wre analyzed with SSPS 13.0. Data are expressed as mean \pm SD. Variances between groups were analyzed using the Student's t-test and one-way analysis of variance (ANOVA). $\mathrm{P}<0.05$ was considered to indicate a statistically significant result.

\section{Results}

Baicalin does not affect the viability of breast cancer cells. Previous studies have revealed that baicalin exerts beneficial effects on inhibiting cell proliferation and inducing apoptosis in various cancer types (24-26). To evaluate whether baicalin affects the viability of breast cancer cells, the crystal violet assay was performed in MDA-MB-231 and 4T1 cells. As shown in Fig. 1B and C, the cell viability had no significant difference when the breast cancer cells were treated with increasing concentrations of baicalin up to $100 \mu \mathrm{M}$. 
A
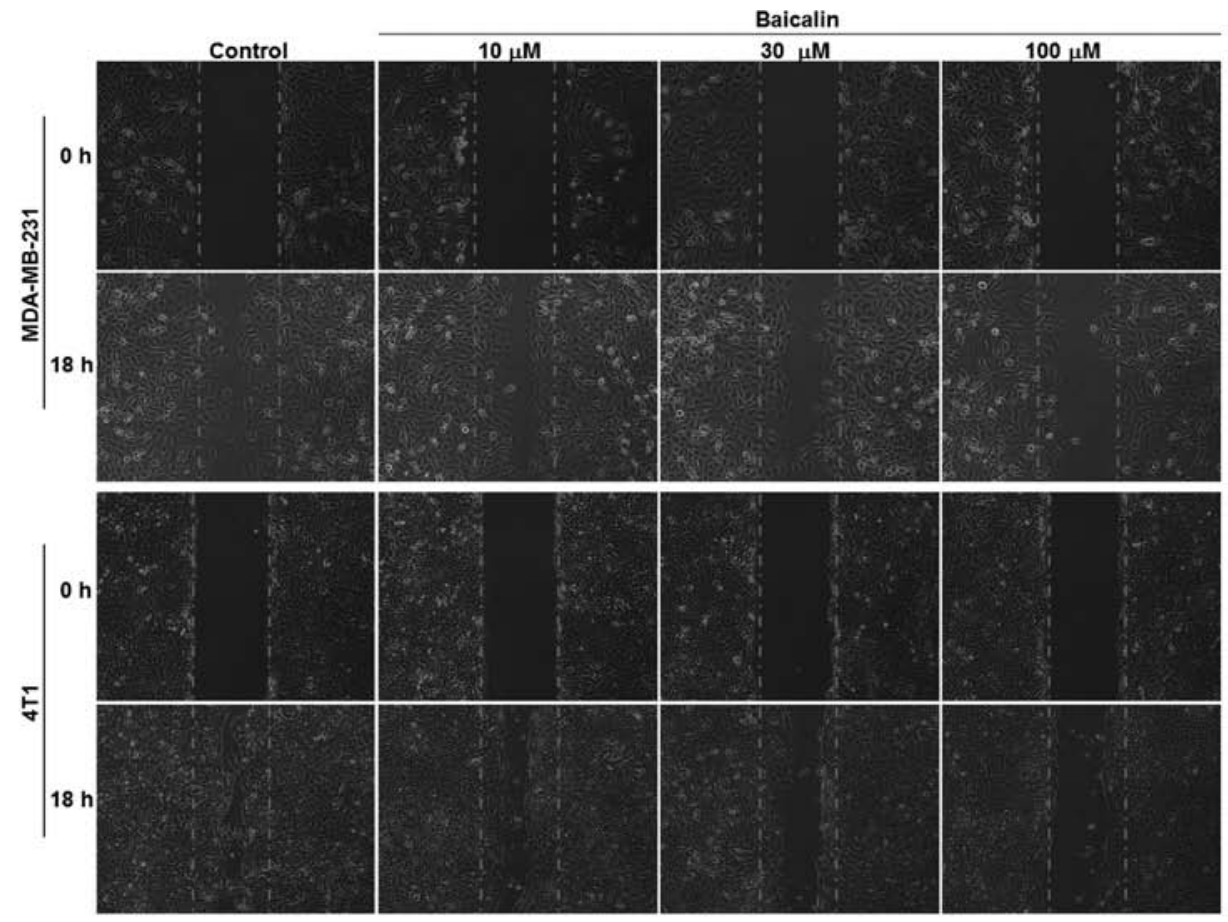

B

MDA-MB-231

$4 \mathrm{~T} 1$
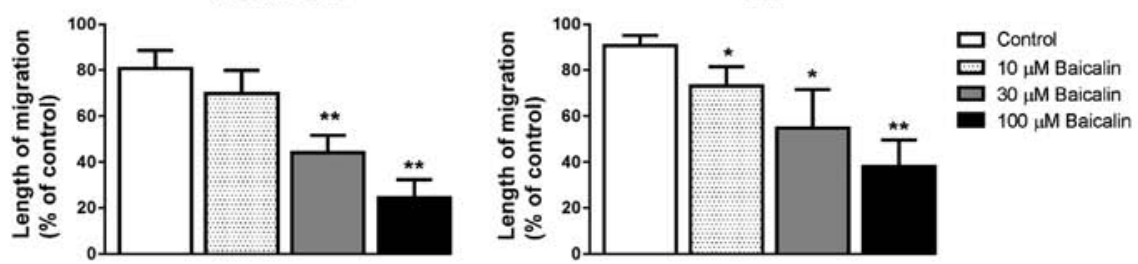

C

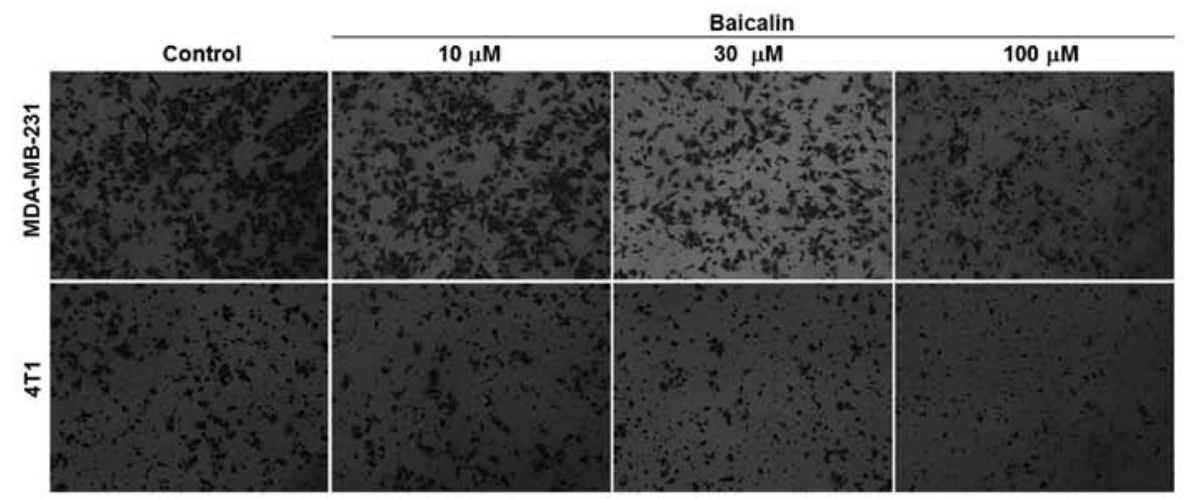

D
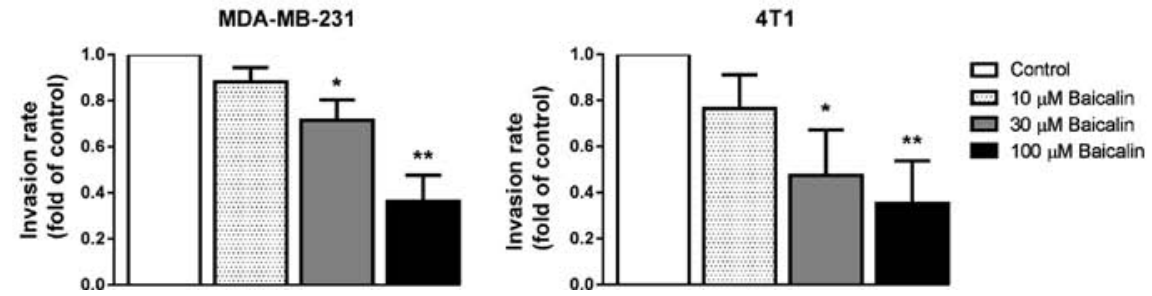

Figure 2. Baicalin suppresses breast cancer cell migration and invasion in vitro. The highly invasive breast cancer cells were pretreated with different concentrations of baicalin for the indicated time. (A) Cell migration was determined by wound healing assay. (B) Quantification of the migrated cells. (C) Cell invasion was determined by Transwell invasion assay. (D) Quantification of the invasive cells. The results are shown as the mean $\pm \mathrm{SD}(\mathrm{n}=3),{ }^{*} \mathrm{P}<0.05,{ }^{* * *} \mathrm{P}<0.01$ compared with the control.

Baicalin suppresses breast cancer cell migration and invasion. To determine whether baicalin has the potential to inhibit breast cancer cell migration and invasion, wound healing and transwell migration assays were performed using two highly aggressive breast cancer cell lines MDA-MB-231 and $4 \mathrm{~T} 1$. In the wound healing assay, following pretreatment 
A

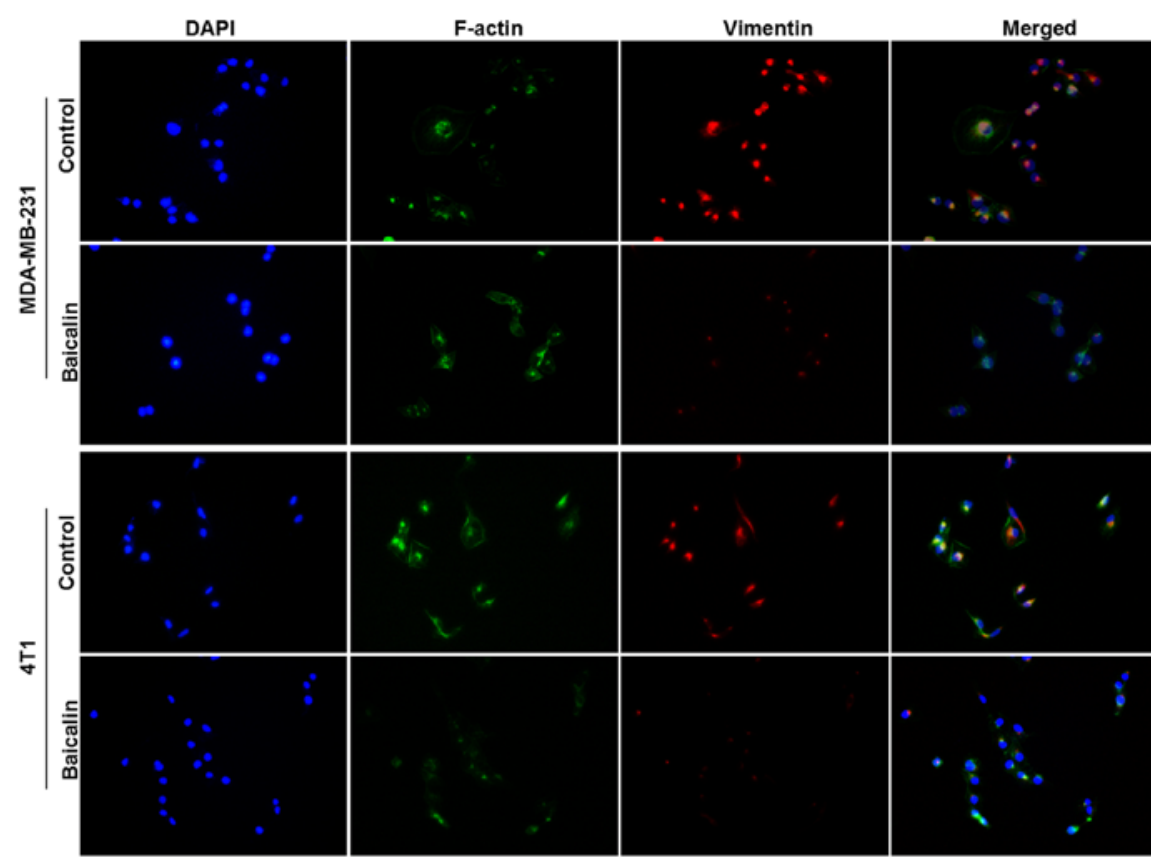

B

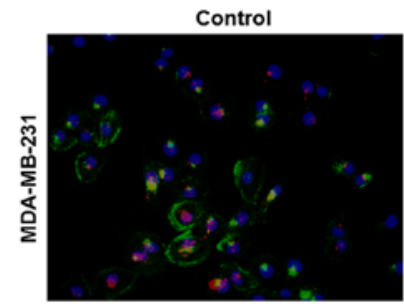

Baicalin

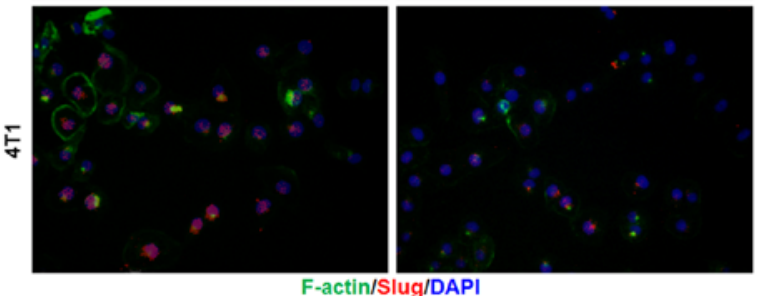

C

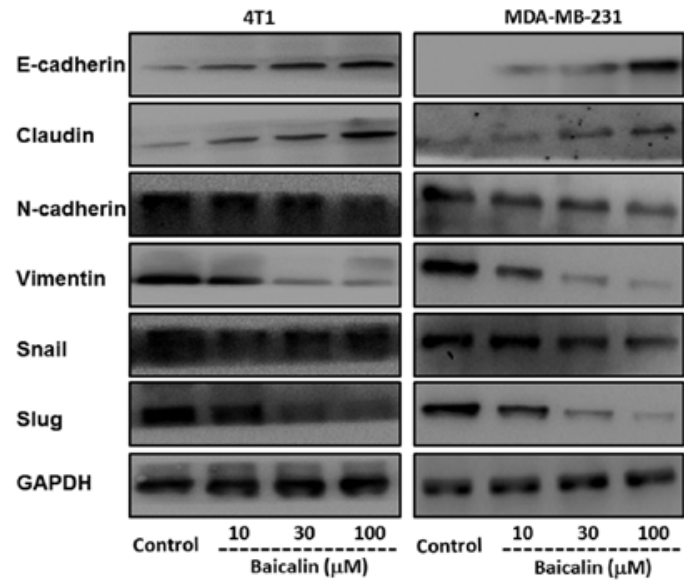

\section{D}

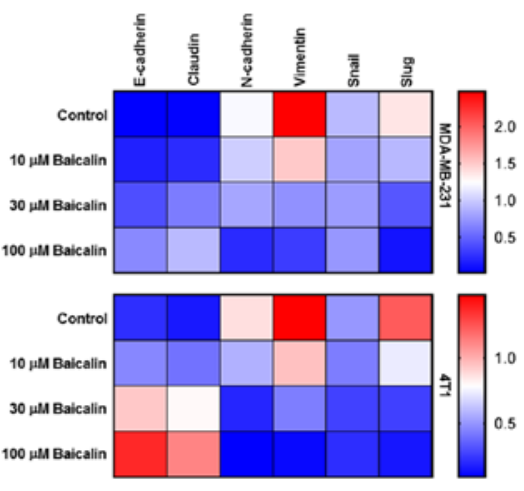

Figure 3. Baicalin reverses epithelial-to-mesenchymal transition (EMT) in breast cancer cells. The highly invasive breast cancer cells were pretreated with different concentrations of baicalin for $24 \mathrm{~h}$. (A) The breast cancer cells were subjected to immunofluorescence assay for vimentin and F-actin. (B) Slug was determined by immunofluorescence assay. (C) The breast cancer cells were subjected to western blot analysis for the indicated proteins. (D) The breast cancer cells were subjected to qRT-PCR for the indicated mRNAs.

with baicalin $(10,30$, or $100 \mu \mathrm{M})$, the wound scratches in the baicalin-treated groups were wider than those noted in the control group at $18 \mathrm{~h}$, and had a dose-dependent increasing trend (Fig. 2A and B), indicating that baicalin inhibited cell migration of breast cancer cells. In the cell invasion experiment by Transwell assay, results similar to those of the wound healing assay were observed in the baicalin-treated breast cancer cells (Fig. 2C and D). 
A
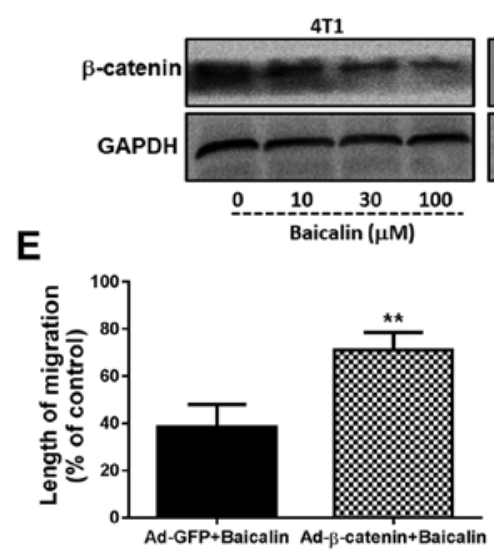

$\mathbf{F}$

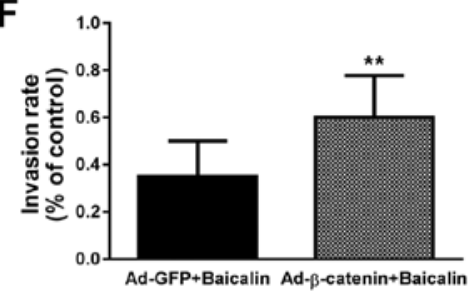

B

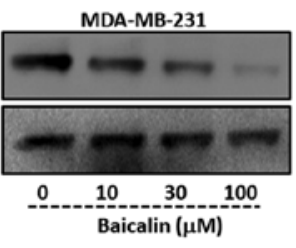

C

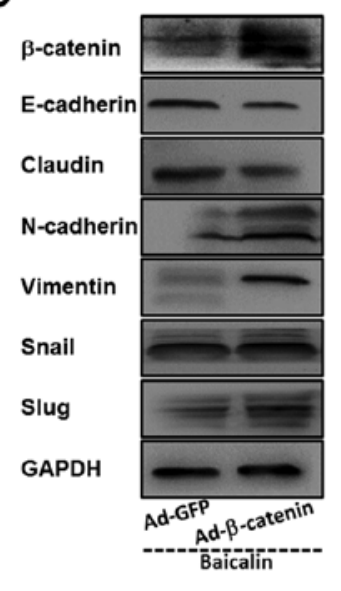

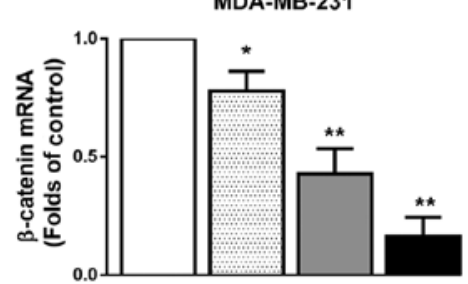
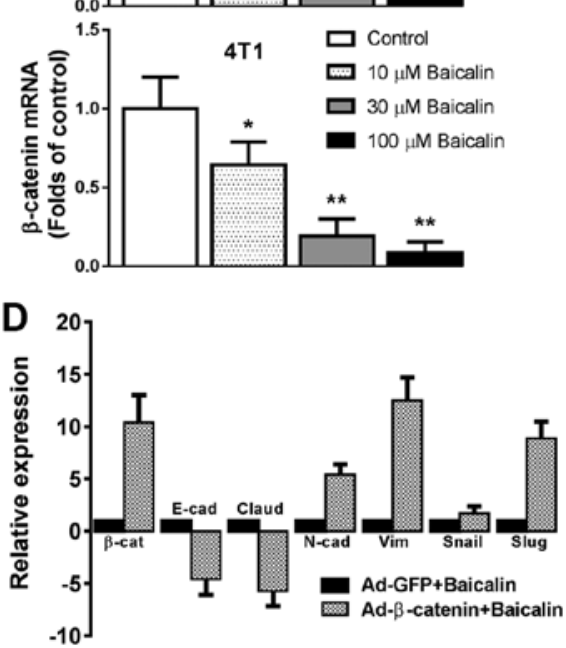

Figure 4. Baicalin downregulates the expression of $\beta$-catenin to mediate its anti-metastatic effect in vitro. The highly invasive breast cancer cells with or without Ad- $\beta$-catenin overexpression were pretreated with different concentrations of baicalin for $24 \mathrm{~h}$. (A) $\beta$-catenin protein was determined by western blotting. (B) $\beta$-catenin mRNA was detected by qRT-PCR. (C) EMT markers and transcriptional factors were determined by western blotting. (D) EMT markers and transcriptional factors were detected by qRT-PCR. (E) Cell migration was determined by wound healing assay. (F) Cell invasion was determined by Transwell invasion assay. The results are shown as the mean $\pm \mathrm{SD}(\mathrm{n}=3),{ }^{*} \mathrm{P}<0.05,{ }^{* *} \mathrm{P}<0.01$. EMT, epithelial-to-mesenchymal transition.

Baicalin reverses breast cancer cell EMT process. It has been confirmed that EMT plays a major role in tumor metastasis. In order to explore the relationship between baicalin and EMT, we used varying concentrations of baicalin to treat MDA-MB-231 and $4 \mathrm{~T} 1$ breast cancer cells, respectively. IF assay indicated that the mesenchymal marker vimentin was degraded and the cytoskeletal protein F-actin was remolded after the two breast cancer cell lines were treated with $100 \mu \mathrm{M}$ baicalin (Fig. 3A), and the expression of Slug, one of the major EMT TFs that is usually maintained at a high level in highly invasive breast cancer cells (27), was downregulated in the baicalin-treated MDA-MB-231 and 4T1 cells (Fig. 3B). Furthermore, western blotting and quantitative RT-PCR also showed that epithelial markers E-cadherin and claudin were upregulated while mesenchymal markers $\mathrm{N}$-cadherin and vimentin and relative TFs Snail and Slug were downregulated in a dose-dependent manner by baicalin in the two highly invasive breast cancer cell lines (Fig. 3C and D).

$\beta$-catenin contributes to the beneficial effects of baicalin in regards to the metastasis and EMT of breast cancer cells. Prior studies have demonstrated that baicalin can inhibit breast cancer cell migration through the p38 MAPK signaling pathway (18). Cancer metastasis and EMT are complex processes which are influenced by numerous signaling pathways through crosstalk (6). In view of the important status of $\beta$-catenin in cancer metastasis and EMT, we first determined whether baicalin affects the $\beta$-catenin signaling pathway. Western blotting and quantitative RT-PCR analysis found that $\beta$-catenin was markedly expressed in the MDA-MB-231 and $4 \mathrm{~T} 1$ breast cancer cells, and pretreatment with baicalin dose-dependently downregulated the expression of $\beta$-catenin protein and mRNA (Fig. 4A and B). In addition, overexpression of $\beta$-catenin in the baicalin-treated $4 \mathrm{~T} 1$ cells by adenovirus vector system, significantly blunted the role of baicalin in regards to the expression levels of EMT-related molecules and TFs, returning cells back to a mesenchymal type (Fig. 4C and D). Simultaneously, the inhibitory effects of baicalin on the migration and invasion of highly invasive breast cancer cells were also reversed with the overexpression of $\beta$-catenin (Fig. 4E and F).

Baicalin suppresses the metastasis of breast cancer cells in vivo. Finally, we constructed a xenograft metastasis tumor model of $4 \mathrm{~T} 1$ breast cancer cells to investigate the effects of baicalin on breast cancer metastasis in vivo. As shown in Fig. 5A and B, we found that the numbers of metastatic nodules on the surface of the liver and lung in the baicalin-treated group were less than these numbers in the control group. H\&E staining of the liver and lung also indicated that baicalin reduced the metastatic lesions of breast cancer cells in the liver and lung tissues (Fig. 5C). Similar to the in vitro experiment, the expression levels of mesenchymal marker vimentin and EMT-activating TF Slug in the orthotopic tumor tissues were downregulated by baicalin (Fig. 5D). Finally, immunohistochemistry showed that baicalin inhibited $\beta$-catenin expression in the orthotopic tumor tissues (Fig. 5E). 
A

B
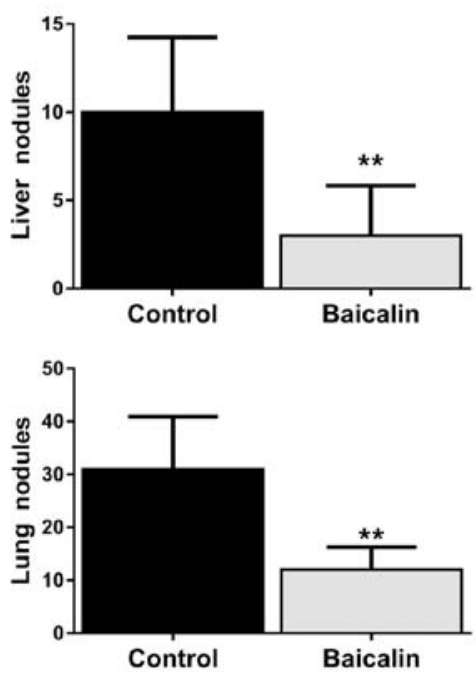

D

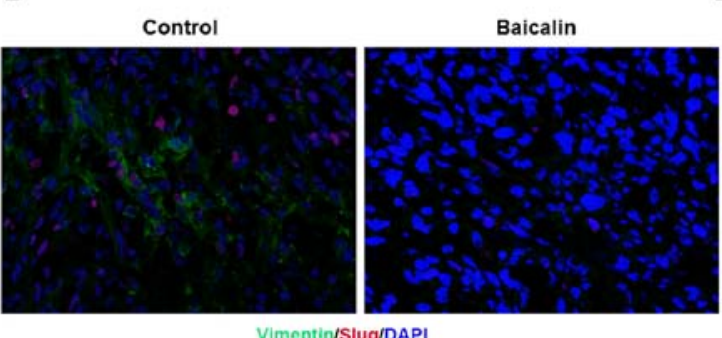

C
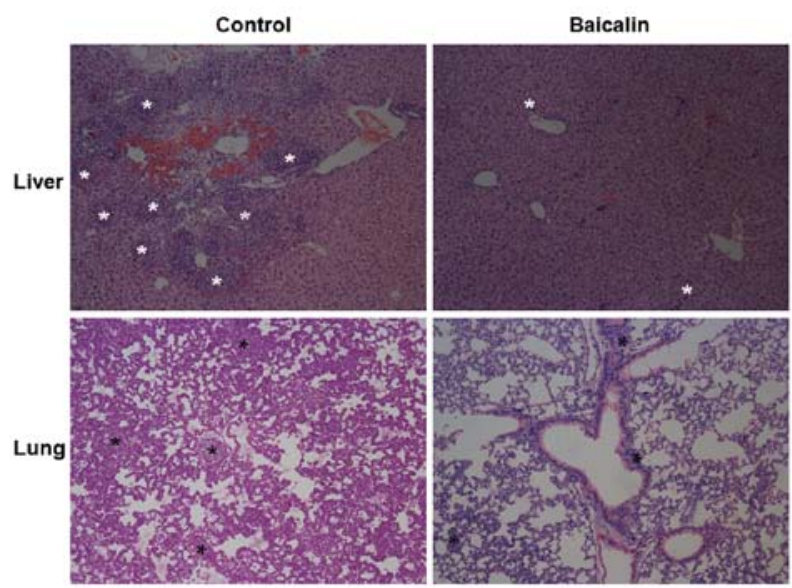

E

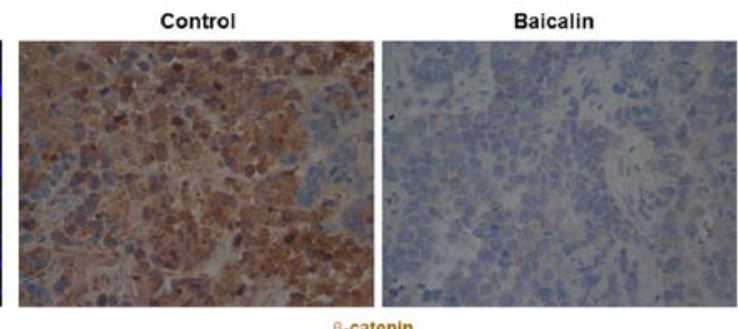

Figure 5. Baicalin inhibits the metastasis of breast cancer in vivo. Female BALB/c mice were injected subcutaneously with $1 \times 10^{6} 4 \mathrm{~T} 1$ cells into the bilateral gluteal region. One week after inoculation, the mice received PBS or $100 \mathrm{mg} / \mathrm{kg}$ baicalin in an intraperitoneal injection approach every 3 days. After 6 weeks, all mice were sacrificed under anesthesia. Tumors, liver and lungs were excised and tumor nodules were counted and tissues were fixed for analysis. (A) Tumor nodules in the liver were counted. (B) Tumor nodules in the lung were counted. (C) The metastatic nodules are shown as asterisks in the images of the H\&Estained sections of the liver and lung. (D) Vimentin and Slug in the primary tumors were examined by immunofluorescence. (E) $\beta$-catenin in the primary tumors was examined by immunohistochemistry. The results are shown as the mean $\pm \mathrm{SD}(\mathrm{n}=5),{ }^{* * *} \mathrm{P}<0.01$.

\section{Discussion}

Although the mortality rate of breast cancer is decreasing along with more efficacious adjuvant treatments and systematic treatment schedules, the mortality rate is still high and breast cancer is the leading cause of cancer-related mortality among middle-age women (28). As is known, the main cause of cancer death is not the primary tumor but its metastasis to distal sites and metastasis-related diseases. Partly due to ineffective therapy or therapeutic resistance, the incidence of metastasis is becoming more and more frequent, which causes a high percentage of recurrence and poor prognosis for patients. In the present study, we investigated the potential effect of baicalin on the inhibition of metastasis in breast cancer cells and elucidated the underlying molecular mechanisms. Our findings revealed that baicalin effectively inhibited the migration and invasion of breast cancer cell lines MDA-MB-231 and 4T1, and suppressed the lung and liver metastasis of breast cancer xenograft tumors derived from 4T1 breast cancer cells, suggesting that baicalin may serve as a rational therapeutic strategy to suppress the metastasis of breast cancer.

A compelling body of evidence has confirmed that EMT is not only a crucial morphogenic process normally activated during embryogenesis and reconstruction of wounded tissues, but also plays an important role during the metastasis of cancers, allowing dynamic and reversible transition from adhesive, non-mobile, epithelial-like phenotype to mobile, invasive mesenchymal-like phenotype, which is utilized by cancer cells to promote their capabilities of local invasion and distant metastasis (29). Indeed, EMT exhibits an essential manifestation of molecular changes, namely loss of epithelial markers E-cadherin and claudin and gain of mesenchymal markers vimentin and $\mathrm{N}$-cadherin (30). Previous studies have elucidated that EMT is associated with clinicopathological features, resistance to apoptosis, evasion of the immune response and poor prognosis of breast cancer (31). Based on its anti-migratory potential, we aimed to ascertain whether baicalin has an influence on EMT in highly aggressive breast cancer cell lines. Our study found that MDA-MB-231 and 4T1 cancer cells acquired epithelial features and lost mesenchymal phenotype at the same time following treatment with baicalin, indicating that baicalin could effectively reverse EMT in highly aggressive breast cancer cells. A large number of previous studies have shown that Slug is an important transcription factor of EMT, and promotes the process of EMT and cancer metastasis $(32,33)$. Thus, we analyzed the expression and nuclear translocation of Slug, and found that the expression and nuclear translocation of Slug was decreased after intervention of baicalin. All these data confirmed that the effect of baicalin on the migration of breast cancer cells resulted from the reversion of EMT. 
To date, numerous studies have demonstrated that multiple signaling pathways participate in the regulation of EMT, cell migration, invasion and tumor metastasis, including the Wnt/ $\beta$-catenin, NF- $\kappa$ B and p38MAPK signaling pathways (18,34-36). Among them, the aberrant activation of the Wnt/ $\beta$-catenin signaling pathway has been found in many human malignancies. In terms of the canonical Wnt/ $\beta$-catenin signaling pathway, $\beta$-catenin is a key component as a TF. Upon Wnt ligand engagement, $\beta$-catenin is activated and disrupted, followed by its translocation from the cytoplasm into the nucleus, where it facilitates the transcription of genes involved in EMT, such as Twist, Snail, Slug, which repress the expression of E-cadherin, influencing cell junction and polarity (37). Previous studies have reported that overexpression and hyperactivation of $\beta$-catenin are observed in aggressive basallike breast cancer, and are positively correlated with poor patient clinical outcome (38-40). Therefore, $\beta$-catenin may be a novel therapeutic target with which to overcome breast cancer metastasis and improve the prognosis of breast cancer patients. In the present study, we found that $\beta$-catenin was robustly expressed in MDA-MB-231 and 4T1 breast cancer cells which was dose-dependently inhibited by baicalin at the protein and mRNA levels. Using a xenograft mouse in vivo model, baicalin markedly downregulated the expression of $\beta$-catenin in primary tumor tissues. Furthermore, overexpression of $\beta$-catenin by an adenovirus vector system markedly blunted the suppressive effects of baicalin on metastasis and EMT in breast cancer cells, suggesting that these beneficial effects of baicalin may be involved in the downregulation of $\beta$-catenin signaling.

In conclusion, our experimental data showed that baicalin exhibited significant effects on the suppression of migration and metastasis in highly aggressive breast cancer both in vitro and in vivo, which may be through reversal of the EMT process and downregulation of $\beta$-catenin expression. Therefore, application of baicalin in conjunction with currently conventional adjuvant treatments may provide a novel therapeutic strategy for patients with metastatic breast cancer.

\section{Acknowledgements}

This research was supported by grants from the National Natural Science Foundation of China (no. 81472475). We thank Dr Jianfei Guo, James Winkle College of Pharmacy, University of Cincinnati, for the discussion and proofreading of the manuscript.

\section{References}

1. Torre LA, Bray F, Siegel RL, Ferlay J, Lortet-Tieulent J and Jemal A: Global cancer statistics, 2012. CA Cancer J Clin 65: 87-108, 2015.

2. Chen W, Zheng R, Baade PD, Zhang S, Zeng H, Bray F, Jemal A, $\mathrm{Yu}$ XQ and He J: Cancer statistics in China, 2015. CA Cancer J Clin 66: 115-132, 2016.

3. Arnedos M, Vicier C, Loi S, Lefebvre C, Michiels S, Bonnefoi H and Andre F: Precision medicine for metastatic breast cancer - limitations and solutions. Nat Rev Clin Oncol 12: 693-704, 2015.

4. Samatov TR, Tonevitsky AG and Schumacher U: Epithelial-mesenchymal transition: Focus on metastatic cascade, alternative splicing, non-coding RNAs and modulating compounds. Mol Cancer 12: 107, 2013.
5. Drasin DJ, Robin TP and Ford HL: Breast cancer epithelial-to-mesenchymal transition: Examining the functional consequences of plasticity. Breast Cancer Res 13: 226, 2011.

6. Takebe N, Warren RQ and Ivy SP: Breast cancer growth and metastasis: Interplay between cancer stem cells, embryonic signaling pathways and epithelial-to-mesenchymal transition. Breast Cancer Res 13: 211, 2011.

7. Pan MH and Ho CT: Chemopreventive effects of natural dietary compounds on cancer development. Chem Soc Rev 37: 2558-2574, 2008.

8. Peng-Fei L, Fu-Gen H, Bin-Bin D, Tian-Sheng D, Xiang-Lin H and Ming-Qin Z: Purification and antioxidant activities of baicalin isolated from the root of huangqin (Scutellaria baicalensis gcorsi). J Food Sci Technol 50: 615-619, 2013.

9. Burnett BP, Jia Q, Zhao Y and Levy RM: A medicinal extract of Scutellaria baicalensis and Acacia catechu acts as a dual inhibitor of cyclooxygenase and 5-lipoxygenase to reduce inflammation. J Med Food 10: 442-451, 2007.

10. Li BQ, Fu T, Gong WH, Dunlop N, Kung H, Yan Y, Kang J and Wang JM: The flavonoid baicalin exhibits anti-inflammatory activity by binding to chemokines. Immunopharmacology 49: 295-306, 2000.

11. Li BQ, Fu T, Dongyan Y, Mikovits JA, Ruscetti FW and Wang JM: Flavonoid baicalin inhibits HIV-1 infection at the level of viral entry. Biochem Biophys Res Commun 276: 534-538, 2000.

12. Shieh DE, Cheng HY, Yen MH, Chiang LC and Lin CC: Baicalin-induced apoptosis is mediated by Bcl-2-dependent, but not p53-dependent, pathway in human leukemia cell lines. Am J Chin Med 34: 245-261, 2006.

13. Chen H, Gao Y, Wu J, Chen Y, Chen B, Hu J and Zhou J: Exploring therapeutic potentials of baicalin and its aglycone baicalein for hematological malignancies. Cancer Lett 354: 5-11, 2014.

14. Yu Y, Pei M and Li L: Baicalin induces apoptosis in hepatic cancer cells in vitro and suppresses tumor growth in vivo. Int J Clin Exp Med 8: 8958-8967, 2015.

15. Shu YJ, Bao RF, Wu XS, Weng H, Ding Q, Cao Y, Li ML, Mu JS, Wu WG, Ding QC, et al: Baicalin induces apoptosis of gallbladder carcinoma cells in vitro via a mitochondrial-mediated pathway and suppresses tumor growth in vivo. Anticancer Agents Med Chem 14: 1136-1145, 2014.

16. Gong WY, Wu JF, Liu BJ, Zhang HY, Cao YX, Sun J, Lv YB, Wu X and Dong JC: Flavonoid components in Scutellaria baicalensis inhibit nicotine-induced proliferation, metastasis and lung cancer-associated inflammation in vitro. Int J Oncol 44: 1561-1570, 2014.

17. Yang BL, Chen HJ, Chen YG, Gu YF, Zhang SP, Lin Q and Sun Y: Inhibitory effects of baicalin on orthotopic xenografts of colorectal cancer cells that are deficient in a mismatch repair gene in nude mice. Int J Colorectal Dis 28: 547-553, 2013.

18. Wang XF, Zhou QM, Du J, Zhang H, Lu YY and Su SB: Baicalin suppresses migration, invasion and metastasis of breast cancer via p38MAPK signaling pathway. Anticancer Agents Med Chem 13: 923-931, 2013.

19. Lin C, Tsai SC, Tseng MT, Peng SF, Kuo SC, Lin MW, Hsu YM, Lee MR, Amagaya S, Huang WW, et al: AKT serine/threonine protein kinase modulates baicalin-triggered autophagy in human bladder cancer T24 cells. Int J Oncol 42: 993-1000, 2013.

20. Gao J, Morgan WA, Sanchez-Medina A and Corcoran O: The ethanol extract of Scutellaria baicalensis and the active compounds induce cell cycle arrest and apoptosis including upregulation of p53 and Bax in human lung cancer cells. Toxicol Appl Pharmacol 254: 221-228, 2011.

21. Wang B, Wan JY, Zhang L and Min S: Synthetic RGDS peptide attenuates mechanical ventilation-induced lung injury in rats. Exp Lung Res 38: 204-210, 2012.

22. Tao Z, Shi A, Lu C, Song T, Zhang Z and Zhao J: Breast cancer: Epidemiology and etiology. Cell Biochem Biophys 72: 333-338, 2015.

23. Pulaski BA and Ostrand-Rosenberg S: Mouse 4T1 breast tumor model. Curr Protoc Immunol: Chapter 20: Unit 20.2, 2001. doi: 10.1002/0471142735.im2002s39.

24. Chen WC, Kuo TH, Tzeng YS and Tsai YC: Baicalin induces apoptosis in SW620 human colorectal carcinoma cells in vitro and suppresses tumor growth in vivo. Molecules 17: 3844-3857, 2012.

25. Dong LH, Wen JK, Miao SB, Jia Z, Hu HJ, Sun RH, Wu Y and Han M: Baicalin inhibits PDGF-BB-stimulated vascular smooth muscle cell proliferation through suppressing PDGFR $\beta$-ERK signaling and increase in p27 accumulation and prevents injury-induced neointimal hyperplasia. Cell Res 20: 1252-1262, 2010. 
26. Huang Y, Hu J, Zheng J, Li J, Wei T, Zheng Z and Chen Y: Down-regulation of the PI3K/Akt signaling pathway and induction of apoptosis in CA46 Burkitt lymphoma cells by baicalin. J Exp Clin Cancer Res 31: 48, 2012.

27. Grzegrzolka J, Biala M, Wojtyra P, Kobierzycki C, Olbromski M, Gomulkiewicz A, Piotrowska A, Rys J, Podhorska-Okolow M and Dziegiel P: Expression of EMT markers SLUG and TWIST in breast cancer. Anticancer Res 35: 3961-3968, 2015.

28. Siegel RL, Miller KD and Jemal A: Cancer statistics, 2016. CA Cancer J Clin 66: 7-30, 2016.

29. Nickel A and Stadler SC: Role of epigenetic mechanisms in epithelial-to-mesenchymal transition of breast cancer cells. Transl Res 165: 126-142, 2015.

30. Thiery JP: Epithelial-mesenchymal transitions in tumour progression. Nat Rev Cancer 2: 442-454, 2002.

31. Bulfoni M, Gerratana L, Del Ben F, Marzinotto S, Sorrentino M, Turetta M, Scoles G, Toffoletto B, Isola M, Beltrami CA, et al: In patients with metastatic breast cancer the identification of circulating tumor cells in epithelial-to-mesenchymal transition is associated with a poor prognosis. Breast Cancer Res 18: 30, 2016

32. Wang LK, Pan SH, Chang YL, Hung PF, Kao SH, Wang WL, Lin CW, Yang SC, Liang CH, Wu CT, et al: MDA-9/Syntenin-Slug transcriptional complex promote epithelial-mesenchymal transition and invasion/metastasis in lung adenocarcinoma. Oncotarget 7: 386-401, 2016

33. Ganesan R, Mallets E and Gomez-Cambronero J: The transcription factors Slug (SNAI2) and Snail (SNAI1) regulate phospholipase D (PLD) promoter in opposite ways towards cancer cell invasion. Mol Oncol 10: 663-676, 2016.

34. Hino M, Kamo M, Saito D, Kyakumoto S, Shibata T, Mizuki $\mathrm{H}$ and Ishisaki A: Transforming growth factor- $\beta 1$ induces invasion ability of HSC-4 human oral squamous cell carcinoma cells through the Slug/Wnt-5b/MMP-10 signalling axis. J Biochem 159: 631-640, 2016.
35. Tsai JH, Hsu LS, Lin CL, Hong HM, Pan MH, Way TD and Chen WJ: 3,5,4'-Trimethoxystilbene, a natural methoxylated analog of resveratrol, inhibits breast cancer cell invasiveness by downregulation of $\mathrm{PI} 3 \mathrm{~K} / \mathrm{Akt}$ and $\mathrm{Wnt} / \beta$-catenin signaling cascades and reversal of epithelial-mesenchymal transition. Toxicol Appl Pharmacol 272: 746-756, 2013.

36. Chung H, Choi HS, Seo EK, Kang DH and Oh ES: Baicalin and baicalein inhibit transforming growth factor- $\beta 1$-mediated epithelial-mesenchymal transition in human breast epithelial cells. Biochem Biophys Res Commun 458: 707-713, 2015.

37. Ghahhari NM and Babashah S: Interplay between microRNAs and $\mathrm{WNT} / \beta$-catenin signalling pathway regulates epithelial-mesenchymal transition in cancer. Eur J Cancer 51: 1638-1649, 2015.

38. Nowicki A, Sporny S and Duda-Szymańska J: $\beta$-catenin as a prognostic factor for prostate cancer (PCa). Cent European J Urol 65: 119-123, 2012.

39. Chien AJ, Moore EC, Lonsdorf AS, Kulikauskas RM, Rothberg BG, Berger AJ, Major MB, Hwang ST, Rimm DL and Moon RT: Activated Wnt/beta-catenin signaling in melanoma is associated with decreased proliferation in patient tumors and a murine melanoma model. Proc Natl Acad Sci USA 106: 1193-1198, 2009.

40. Cuello-Carrión FD, Shortrede JE, Alvarez-Olmedo D, Cayado-Gutiérrez N, Castro GN, Zoppino FC, Guerrero M, Martinis E, Wuilloud R, Gómez NN, et al: HER2 and $\beta$-catenin protein location: Importance in the prognosis of breast cancer patients and their correlation when breast cancer cells suffer stressful situations. Clin Exp Metastasis 32: 151-168, 2015. 